\title{
MAXIMAL INDEPENDENT COLLECTIONS OF CLOSED SETS
}

\author{
HARVY LEE BAKER, JR.
}

\begin{abstract}
A theorem is proved which implies that if $X$ is a separable metric space then there exists a countable maximal independent subset of the lattice of closed subsets of $X$. In the case where $X$ has no isolated points this independent set is nontrivial in the sense that $X$ does not belong to it and it contains no singletons. Furthermore, if $X$ is a compact metric continuum such that $\bigcup\left\{O \mid O\right.$ is an open subset of $X$ and $O$ is homeomorphic to $E^{n}$ for some $n>1\}$ is dense in $X$ then there exists a countable maximal such collection whose elements are connected. This complements previous work by the author which characterized continua for which there are such collections of a specialized nature.
\end{abstract}

1. An independent subset of a partially ordered set is one such that no two elements of it are comparable. See [5]. An amonotonic collection of sets is one which is independent relative to set inclusion. A complete amonotonic decomposition of a connected $T_{1}$ space $T$ is a maximal amonotonic collection of closed connected subsets of $T$ which is nontrivial in the sense that it contains at least two elements and at least one element of it is not a singleton.

No compact metric continuum has a finite complete amonotonic decomposition (Theorem 1.1 of [3]), whereas many have countable such decompositions and some have only uncountable ones (arcs, indecomposable continua, etc.). Theorem 2.3 is the main object in the present paper and describes maximal independent subsets of the lattice of closed subsets of many $T_{3}$ spaces (all those having "pseudodevelopments"). The cardinality of the maximal independent subset obtained by use of this theorem is often less than the cardinality of the topology of the space, and in particular it follows that if $X$ is a separable metric nondegenerate space then there is a countable maximal independent subset of the lattice of closed subsets of $X$ which is nontrivial in the sense that it does not contain $X$, and if the set of isolated points of $X$ is not dense in $X$ then the only singletons belonging to it are isolated points. Theorem 2.3 also implies that if $T$

Received by the editors January $20,1970$.

AMS 1969 subject classifications. Primary 5440; Secondary 5455, 5425.

Key words and phrases. Independent subset of a partially ordered set, amonotonic collection of sets, complete amonotonic decomposition of a continuum, pseudodevelopment of a space.

(c) American Mathematical Society 1972 
is a compact metric continuum and

$$
\bigcup\left\{O \mid O \text { is open in } T \text { and } O \cong E^{n} \text { for some } n \geqq 2\right\}
$$

is dense in $T$ then there exists a countable complete amonotonic decomposition of $T$. An even larger class of continua which have countable complete amonotonic decompositions is given in Theorem 2.5. Even this theorem, however, falls short of characterizing those continua which do have such decompositions. For example in [3] the following statements concerning a compact metric continuum $M$ are shown to be equivalent: (A) There is a complete amonotonic decomposition $G$ of $M$ such that if $g_{1}$ and $g_{2}$ are distinct elements of $G$ then $M=g_{1} \cup g_{2}$, and $M-g_{1}$ is connected. (B) There is a monotone continuous mapping $F$ from $M$ onto a simple closed curve $J$ such that if $H$ is a proper closed subset of $M$ then $F(H) \neq J$. It is impossible to obtain a countable complete amonotonic decomposition of such an $M$ using Theorem 2.5 (note that statement (A) implies that $G$ is countable). Finite graphs with no end points, and continua related to them the way $M$ is related to $S^{1}$ in statement (B), also have countable complete amonotonic decompositions (see [4] and [2]), but no such decomposition can be obtained by application of Theorem 2.5 .

It is of interest to note that the complete amonotonic decompositions obtained in [4] for finite graphs with no end points, and the decomposition $G$ obtained in statement (A), are not maximal independent subsets of the lattice of closed subsets of the respective spaces, but that the decompositions obtained in Theorem 2.5 and Theorem 2.6 (for "near manifolds" $T$ ) are indeed maximal in this lattice.

2. The following definitions introduce terminology used in the statement of Theorem 2.3 and throughout the proof of it.

DeFINITION 2.1. A pseudodevelopment of the $T_{3}$ space $(X, T)$ is a sequence $G=G_{1}, G_{2}, \cdots$ of nonempty collections of disjoint open sets such that (a) for each $n, G_{n+1}$ refines $G_{n}$ and $\mathrm{Cl}\left(G_{n}^{*}\right)=X$ (the star is the union), and (b) if $O$ is a nonempty open set then there is an $n$ such that some element of $G_{n}$ is a subset of $O$.

Definition 2.2. If $G=G_{1}, G_{2}, \cdots$ is a pseudodevelopment of the $T_{3}$ space $(X, T)$ then $W(G)$ will denote the collection to which $X$ belongs if and only if there exists a positive integer $j$ and $j$ distinct elements $X_{1}, X_{2}, \cdots, X_{j}$ of $G_{j}$ such that (a) $X=$ Interior $\left(\bar{X}_{1} \cup \bar{X}_{2} \cup \cdots \cup \bar{X}_{j}\right)$, and (b) if $i$ is an integer such that $1 \leqq i<j$ then $X_{1} \cup X_{2} \cup \cdots \cup X_{j}$ is not a subset of the union of $i$ or fewer elements of $G_{i}$.

THEOREM 2.3. If $G=G_{1}, G_{2}, \cdots$ is a pseudodevelopment of the $T_{3}$ space $(X, T)$ and for each $n$, each element of $G_{n}$ contains at least two elements of $G_{n+1}$, then $W(G)$ is a maximal independent subset of $T$. 
Proof. If for each $n$ we let $W_{n}$ denote the sets of elements of $W(G)$ which are determined by $n$ distinct elements of $G_{n}$, then a routine argument shows that $W_{1}$ is an amonotonic collection and that if $W_{1} \cup W_{2} \cup \cdots \cup W_{i}$ is an amonotonic collection then so is $W_{1} \cup W_{2} \cup \cdots \cup W_{i+1}$. Hence $W(G)$ is an amonotonic collection.

It must be shown now that every open set either contains or is a subset of an element of $W(G)$. Suppose $D$ is an open set. Suppose there exists an integer $i$ such that $D$ intersects at most $i$ elements of $G_{i}$. We will show that in this case $D$ is a subset of some set of $W(G)$. Let $O_{1}, O_{2}, \cdots, O_{n}$ denote the distinct elements of $G_{i}$ which intersect $D$. We note that since, for each $i$, the elements of $G_{i}$ are disjoint and $\mathrm{Cl}\left(G_{i}^{*}\right)=X$, the set $I=$ Interior $\left(\bar{O}_{1} \cup \bar{O}_{2} \cup \cdots \cup \bar{O}_{n}\right)$ must contain $D$. Hence if we can establish that $I$ is subset of a set of $W(G)$ we are through with this portion of the argument.

That this is so follows from the seemingly more general proposition: If $D_{1}, D_{2}, \cdots, D_{m}$ are distinct elements of $G_{h}$ where $m \leqq h$ then Interior $\left(\bar{D}_{1} \cup \bar{D}_{2} \cup \cdots \cup \bar{D}_{m}\right)$ is a subset of a set of $W(G)$. We prove this proposition by letting $e$ denote the least positive integer such that $D_{1} \cup D_{2} \cup \cdots \cup D_{m}$ is a subset of $e$ or fewer elements of $G_{e}$. We know however that $G_{e}$ contains at least $2^{e-1}$, hence at least $e$, elements. Thus we can find $e$ distinct elements $h_{1}, h_{2}, \ldots, h_{e}$ of $G_{e}$ such that $D_{1} \cup D_{2} \cup \cdots \cup D_{m} \subset h_{1} \cup h_{2} \cup \cdots \cup h_{e}$. Now $h_{1} \cup h_{2} \cup \cdots \cup h_{e}$ satisfies (a) of Definition 2.2, and if (b) is not satisfied then there is an $l$ such that $1 \leqq l<e$ and $h_{1} \cup h_{2} \cup \cdots \cup h_{e}$ is a subset of the union of $l$ distinct elements of $G_{l}$. But in this case $e$ is no longer the least positive integer such that $D_{1} \cup D_{2} \cup \cdots \cup D_{m}$ is a subset of $e$ or fewer elements of $G_{e}$, a contradiction. Hence Int $\left(\bar{h}_{1} \cup \bar{h}_{2} \cup \cdots \cup \bar{h}_{e}\right) \in W(G)$, and since $D_{1} \cup D_{2} \cup \cdots \cup D_{m} \subset h_{1} \cup h_{2} \cup \cdots \cup h_{e}$ implies

$$
\text { Int }\left(\bar{D}_{1} \cup \bar{D}_{2} \cup \cdots \cup \bar{D}_{m}\right) \subset \operatorname{Int}\left(\bar{h}_{1} \cup \bar{h}_{2} \cup \cdots \cup \bar{h}_{e}\right) \text {, }
$$

we are through.

Suppose now that for each $i, D$ intersects at least $i+1$ elements of $G_{i}$. We will show that $D$ contains a set of $W(G)$. There exist an integer $k$ and two distinct elements $X_{1}$ and $X_{2}$ of $G_{k}$ such that $\bar{X}_{1} \cup \bar{X}_{2} \subset D$ and $X_{1}$ and $X_{2}$ lie in the same element of $G_{k-1}$. Let $h_{1}^{1}$ denote the element of $G_{1}$ which contains $\bar{X}_{1} \cup \bar{X}_{2}$ and let $h_{1}^{2}$ be an element of $G_{1}$ distinct from $h_{1}^{1}$ which also intersects $D$. Now let $h_{2}^{1}$ denote the element of $G_{2}$ which lies in $h_{1}^{1}$ and contains $\bar{X}_{1} \cup \bar{X}_{2}$, let $h_{2}^{2}$ denote an element of $G_{2}$ which lies in $h_{1}^{2}$ and intersects $D$, and let $h_{2}^{3}$ denote any element of $G_{2}$ which intersects $D$ and is distinct from $h_{2}^{1}$ and $h_{2}^{2}$. We proceed by choosing four distinct elements $h_{3}^{1}, h_{3}^{2}, h_{3}^{3}, h_{3}^{4}$ of $G_{3}$ such that each intersects $D$ and such that $h_{3}^{1} \subset h_{2}^{1}$, $h_{3}^{2} \subset h_{2}^{2}, h_{3}^{3} \subset h_{2}^{3}$ and $\bar{X}_{1} \cup \bar{X}_{2} \subset h_{3}^{1}$. We continue in this manner until we 
finally obtain $k$ distinct elements $h_{k-1}^{1}, h_{k-1}^{2}, \cdots, h_{k-1}^{k}$ of $G_{k-1}$ such that each intersects $D, \bar{X}_{1} \cup \bar{X}_{2} \subset h_{k-1}^{1}$ and $h_{k-1}^{a} \subset h_{k-2}^{a}$ for $a=1,2, \cdots$, $k-1$. Notice that the entire set of $h_{i}^{j}$,s has been constructed in such a way that $h_{a}^{b} \subset h_{c}^{b}$ if $a \geqq c$ and $b \leqq c+1$. A consequence of this (one that will be used later) is that at the $(n+1)$ th stage of the construction, $h_{n+1}^{1}, h_{n+1}^{2}, \cdots, h_{n+1}^{n+2}$ are chosen in such a way that the union of all of them does not lie in the union of $n$ elements of $G_{n}$. This implies directly that if $m<n$ then $\bigcup h_{n+1}^{i}$ is not a subset of the union of $m$ or fewer elements of $G_{m}$.

Since $(X, T)$ is $T_{3}$ and $G$ is a pseudodevelopment of $(X, T)$, there is an integer $d>k$ such that for every $a$, where $2 \leqq a \leqq k, h_{k-1}^{a} \cap D$ contains the closure of an element of $G_{d}$. Let $g_{a}$, for $a=2,3, \cdots, k$, be an element of $G_{d}$ whose closure lies in $D \cap h_{k-1}^{a}$. Choose now distinct elements $g_{1}^{1}, g_{1}^{2}, \cdots, g_{1}^{d+1-k}$ of $G_{d}$ such that $g_{1}^{1} \cup g_{1}^{2} \cup \cdots \cup g_{1}^{d+1-k} \subset X_{1} \cup X_{2}$ and such that if $k+1 \leqq i \leqq d$ then no two $g_{1}^{n}$ 's lie in the same element of $G_{i}$. That this can be done is evident from the facts that $X_{1}$ and $X_{2}$ contain distinct elements $x_{11}, x_{12}$, and $x_{21}, x_{22}$ of $G_{k+1}$ respectively, that $x_{11}, x_{12}$, $x_{21}, x_{22}$ contain distinct elements $x_{111}, x_{112}$, etc. of $G_{k+2}$, and so forth. We could if we wished choose $2^{d-k} g_{1}^{n}$ 's meeting the above requirements.

Let $I=g_{1}^{1} \cup g_{1}^{2} \cup \cdots \cup g_{1}^{d+1-k} \cup g_{2} \cup g_{3} \cup \cdots \cup g_{k}$. The closure of each of the $g$ 's is in $D$, so Interior $I \subset D$. It will be shown now that Interior $I \in W(G)$. Suppose $1 \leqq e<d$ and that $I$ is a subset of the union of $e$ distinct elements of $G_{e}$. If $k-1 \leqq e<d$, then since the $h_{k-1}^{i}$ 's are distinct and $g_{a} \subset h_{k-1}^{a}$ for $a \geqq 2$, it follows that the set

$$
g_{1}^{1} \cup g_{1}^{2} \cup \cdots \cup g_{1}^{d+1-k}
$$

lies in the union of $e-(k-1)=e+1-k$ elements of $G_{e}$. But $e<d$ and, therefore, at least two of the elements $g_{1}^{1}, g_{1}^{2}, \cdots, g_{1}^{d+1-k}$ lie in the same element of $G_{e}$. This contradicts one of the defining properties of the $g_{1}^{i}$ 's as given in the last paragraph. Hence, $1 \leqq e<k-1$. In this case, since $\left\{h_{k-1}^{1}, h_{k-1}^{2}, \cdots, h_{k-1}^{k}\right\}$ properly covers $I$, it follows that

$$
h_{k-1}^{1} \cup h_{k-1}^{2} \cup \cdots \cup h_{k-1}^{k}
$$

is a subset of the union of $e$ elements of $G_{e}$ where $e<k-1$. But this contradicts the concluding three statements of the third paragraph of this proof (one of which in effect says that this is one of the properties inherited by the $h_{k-1}^{i}$ 's as a result of the manner in which they were selected). Therefore Interior $I \subset D$ and Interior $I \in W(G)$, completing the argument.

It is a consequence of the argument above that if $D$ is not a subset of any element of $W(G)$ then it contains infinitely many elements of $W(G)$. This follows since either $d$ or $k$ can be replaced by any larger integer. 
Corresponding to every maximal independent subset $W$ of the topology $T$ on $X$ is a maximal independent subset $W^{\prime}=\{X-w \mid w \in W\}$ of the lattice of closed subsets of $X$. This correspondence gives us the following result.

THEOREM 2.4. If $M$ is a separable metric space, then there exists a countable maximal independent subset $C$ of the lattice of closed subsets of $M$. In the case where $M$ contains no isolated points (i.e. every point of $M$ is a limit point of $M) C$ is nontrivial in the sense that $M \notin C$ and $C$ contains no singletons.

Proof. If $M$ is a separable metric space with no isolated points then it is easy to construct pseudodevelopments of $M$ which satisfy the hypothesis of Theorem 2.3. For example: For every open set $O$ of $M$ let $G(O)$ denote a maximal collection of disjoint open subsets of $O$ whose diameters are less than one half the diameter of $O$. The sequence defined by $G_{1}=G(M), G_{n+1}=\bigcup\left\{G(x) \mid x \in G_{n}\right\}$ is a pseudodevelopment of $M$ satisfying the conditions of Theorem 2.3. Hence there is as a consequence of the remark preceding the statement of this theorem, a maximal independent subset of the lattice of closed subsets of $M$ which has the same cardinality as $W(G)$, where $G=G_{1}, G_{2}, \cdots$. But $W(G)$ is countable since it is a subset of the set of all finite subsets of $\bigcup G_{n}$, and $G_{i}$ is countable for each $i$ since $M$ is separable and the elements of $G_{i}$ are disjoint open sets.

If $M$ has an isolated point $P$ then $\{M-P,\{P\}\}$ is a maximal independent collection of closed subsets of $M$. If $M_{2}=M-\bar{M}_{1}$ is nonempty, where $M_{1}$ is the set of all isolated points of $M$, then there is a nontrivial countable maximal independent subset of the lattice of closed subsets of $M$ of the form $W_{1} \cup\{\{P\} \mid P$ is an isolated point of $M\}$, where $W_{1}$ is a maximal independent collection of closed subsets of $M_{2}$ obtained by application of the results of the first part of this argument.

THeOREM 2.5. If $M$ is a separable metric space, $G=G_{1}, G_{2}, \cdots$ is a pseudodevelopment of $M$ satisfying the hypothesis of Theorem 2.3, and $M-A^{*}$ is connected for every subcollection $A$ of $\cup G_{n}$, then $W^{\prime}=$ $\{M-x \mid x \in W(G)\}$ is a countable complete amonotonic decomposition of $M$. Furthermore, $W^{\prime}$ is also a maximal independent subset of the lattice of closed subsets of $M$.

THEOREM 2.6. If $T$ is a compact metric continuum and $\bigcup_{n>1} T_{n}$ is dense in $T$, where $T_{n}$ is the union of all open subsets of $T$ which are homeomorphic to $E^{n}$, then there exists a countable complete amonotonic decomposition of $T$ which is also a maximal independent collection of closed subsets of $T$. 
Proof. The space $\bigcup_{n>1} T_{n}$ is a separable metric space and has as a basis the collection of all open cells $g$ such that (a) $\bar{g} \subset T_{n}$ for some $n$, (b) the diameter of $g$ is less than the distance from $g$ to $T-\bigcup_{n>1} T_{n}$.

Open cell here is an open subset of $T$ which is homeomorphic to $E^{n}$ for some $n$ and whose closure is a closed cell. From this basis (or any other basis) a pseudodevelopment $G=G_{1}, G_{2}, \cdots$ can be constructed such that the boundaries of the elements of $\bigcup G_{n}$ are disjoint. Now $W(G)$ is a maximal independent subset of the topology of $\bigcup_{n>1} T_{n}$ and, by condition (b) and the fact that $\mathrm{Cl}\left(\bigcup_{n>1} T_{n}\right)=T$, we know that every open set of $T$ which intersects $T-\bigcup_{n>1} T_{n}$ contains an element of $G_{1}$ and hence an element of $W(G)$. Here we should note that Int $\vec{g}=g$ for open cells $g$.

Thus $W(G)$ is a maximal independent subset of the topology on $T$ and the set of complements of the elements of $W(G)$ is a countable maximal independent subset of the lattice of closed subsets of $T$. The elements of $W(G)$ have connected complements since the components of $\bigcup_{n>1} T_{n}$ are connected manifolds of dimension greater than one, no such manifold is disconnected by the union of a finite number of open cells whose boundaries are disjoint, and no element of $\bigcup G_{n}$ has a boundary which intersects $T-\bigcup_{n>1} T_{n}$.

\section{REFERENCES}

1. H. L. Baker, Jr., Complete amonotonic collections of subcontinua of a compact continuum, Notices Amer. Math. Soc. 12 (1965), 91. Abstract \#619-119.

2. - Concerning complete amonotonic collections of subcontinua of a compact continuum, Notices Amer. Math. Soc. 12 (1965), 697. Abstract \#626-24.

3. - Complete amonotonic decompositions of compact continua, Proc. Amer. Math. Soc. 19 (1968), 847-853. MR 38 \#678.

4. - Amonotonic decomposition of finite graphs, Pacific J. Math. (submitted).

5. R. P. Dilworth, A decomposition theorem for partially ordered sets, Ann. of Math. (2) 51 (1950), 161-166. MR 11, 309.

Department of Mathematics, University of Nebraska, Lincoln, Nebraska 68508 